\title{
PTEN expression is involved in the invasive properties of HNSCC: A key protein to consider in locoregional recurrence
}

\author{
JIHANE MRIOUAH ${ }^{1,2}$, CÉDRIC BOURA $^{1,2}$, MYRIEM GARGOURI $^{1,2}$, \\ FRANÇOIS PLÉNAT ${ }^{1,2,3}$ and BÉATRICE FAIVRE ${ }^{1,2}$ \\ ${ }^{1}$ Université de Lorraine, CRAN, UMR 7039, Campus Science, BP 70239; ${ }^{2}$ CNRS, CRAN, UMR 7039; \\ ${ }^{3} \mathrm{CHU}$ Nancy, Service d'Anatomie Pathologique, Vandoeuvre-lès-Nancy 54505, France
}

Received August 19, 2013; Accepted October 11, 2013

DOI: 10.3892/ijo.2013.2219

\begin{abstract}
Specific phenotypic effects of PTEN in head and neck squamous cell carcinoma (HNSCC) remain poorly defined without a direct causal connection between the loss of PTEN function and the progression of cancer. Here, we describe a potential role for PTEN in cancer progression. Using an shRNA targeting PTEN in HNSCC cells, we show that the loss of PTEN expression is associated with a decrease of cell adhesion, a reduction in E-cadherin expression while cell migration is promoted. Together with the tissue organization and molecular markers expressed in tumors derived from shPTEN cells in vivo, this study indicates that HNSCC cells deficient in PTEN expression undergo an epithelial-mesenchymal transition (EMT). Additionally, our results suggest that both the low levels of expression and subcellular localization of PTEN are involved in the EMT phenotype, and ultimately in possible locoregional reccurences. We hypothesize that the loss of PTEN expression as well as the subcellular localization could be of interest as a predictive marker of recurrence in HNSCC.
\end{abstract}

\section{Introduction}

Head and neck squamous cell carcinoma (HNSCC) is one of the most common types of human cancer (1). Only 40-50\% of patients with HNSCC will survive for 5 years after diagnosis and treatment. Despite the advent and use of targeted drugs in the recent past, the survival has not markedly improved. Most patients develop locoregional recurrence, distant metastases or second primary tumors. The underlying cellular and molecular mechanisms that contribute to the initiation and progression of HNSCC have not been completely delineated

Correspondence to: Dr Cédric Boura, Université de Lorraine, CRAN UMR CNRS 7039, Faculté de Médecine, 9 av. de la Forêt de Haye, Bât D, 54505 Vandoeuvre-lès-Nancy, France

E-mail: cedric.boura@univ-lorraine.fr

Key words: PTEN, head and neck squamous cell carcinoma, epithelial-mesenchymal transition, cell adhesion, E-cadherin
(2). Recent advances in the understanding of the oncogenesis of HNSCC have revealed multiple molecular deregulations, with PTEN/PI3K/Akt/mTOR being the most frequently altered signaling pathways. Loss of PTEN expression (phosphatase and tensin homolog deleted on chromosome 10) was found in $29 \%$ of tongue cancers and loss of heterozygosity identified in $40 \%$ of HNSCC (3-5). A decrease in PTEN expression is sufficient to induce tumor progression $(6,7)$. PTEN converts the phosphatidylinositol-3,4,5-trisphosphate $\left(\mathrm{PIP}_{3}\right)$ in the cytoplasm to phosphatidylinositol-4,5-bisphosphate $\left(\mathrm{PIP}_{2}\right)$, thereby directly antagonizing the activity of PI3K (8). Its inactivation is common in cancer and results in constitutive activation of the PI3K/AKT pathway. PTEN also inhibits SHC phosphorylation, reducing the activation of Ras/MAP-kinase (MAPK) pathway (9). Nevertheless, more attention has been focused on its capacity to antagonize the growth and proliferation-promoting actions of PI3K and AKT.

More recently, the protein phosphatase activity of PTEN has received increased scrutiny in aberrant cellular processes, particularly regarding cell motility, invasion and migration. Indeed, it has been demonstrated that PTEN modulates cell migration and invasion by negatively regulating the signals generated at the focal adhesions, through direct dephosphorylation and inhibition of FAK $(10,11)$. Furthermore, PTEN can modulate integrin-mediated focal adhesion formation and cytoskeletal reorganization (12).

The effects of loss of PTEN have primarily been evaluated in cancer cell lines that have other oncogenic mutations and have lost their epithelial phenotype (13). Such an approach has made it difficult to determine the phenotypes directly conferred by the loss of PTEN and to define the stages of tumorigenesis that are specifically influenced by the PTEN loss. To further characterize the role of PTEN in HNSCC carcinogenesis, we have selected two cell clones expressing a small hairpin RNA targeting a specific sequence of PTEN previously validated (14) and the invasive ability of these two cell clones was evaluated both in vitro and in vivo. Our results showed that the tumors originating from PTEN deficient cells have a molecular signature and a phenotype suggesting an undergoing epithelial to mesenchymal transition (EMT). To our knowledge, our study is the first to investigate a role of PTEN in the EMT in HNSCC. 


\section{Materials and methods}

Cell culture and transfections. CAL 27 cells, a human head and neck squamous cell carcinoma (HNSCC) cell line, were used (CRL-2095 ${ }^{\mathrm{TM}}$, LGC Standards, Molsheim, France). The cell line was cultured in phenol-red free RPMI-1640 (Invitrogen, Saint Aubin, France) supplemented with $10 \%$ of heat-inactivated fetal calf serum (Sigma-Aldrich, Saint-Quentin Fallavier, France) at $37^{\circ} \mathrm{C}$ in a humidified atmosphere of $5 \% \mathrm{CO}_{2}$ in air. pSIREN-RetroQ expression vectors containing shPTEN and a pSIREN-RetroQ scramble shRNA vector were transfected in CAL 27 using Jet-PEI (Polyplus Transfection, Illkirch, France) according to the manufacturer's protocol. Stable, pooled populations were then generated under puromycin selection $(0.5 \mu \mathrm{g} / \mathrm{ml}$; Sigma-Aldrich) and clones were isolated by limiting dilution. Two transfected CAL 27 cell clones named shPTEN1 and shPTEN2 were used in this study and compared to scramble vector-transfected cells (shCTL) or parental CAL 27 cells.

Cell proliferation. The effect of PTEN inhibition on cell proliferation was evaluated by trypan blue exclusion assay. Briefly, cells were cultured in 6 -well dishes $\left(2 \times 10^{4}\right.$ cells/well) over 10 days. After the set time, cells were detached using a $0.25 \%$ trypsin-EDTA solution (Sigma) for $7 \mathrm{~min}$ at $37^{\circ} \mathrm{C}$ and resuspended in complete medium. Cells were then stained with $0.4 \%$ (w/v) trypan blue solution and viable cells were counted twice using a Malassez hemocytometer.

Cell adhesion and invasion. For cell adhesion, cells were stained with Hoechst 3342 for $30 \mathrm{~min}$ at $37^{\circ} \mathrm{C}$ before trypsination. Then cells were plated at a density of $2 \times 10^{5}$ cells/wells on fibronectin, collagen I, laminin and vitronectin coated wells (at a concentration of 10, 20,4 and $0.2 \mu \mathrm{g} /$ well, respectively). Cells were allowed to attach for 0.5 and $4 \mathrm{~h}$. Then, the medium containing non-adherent cells was gently removed. Cell adhesion was measured by fluorescence intensity with a microplate reader at $460 \mathrm{~nm}$ (Twinkle LB 970, Berthold Technologies, Thoiry, France).

Cell invasion assay was performed in modified Boyden Chambers with $8-\mu \mathrm{m}$ pore filter inserts (BD Falcon, Erembodegem, Belgium) coated with Matrigel ${ }^{\mathrm{TM}}$ (BD Falcon). Cell lines were added at $2 \times 10^{5}$ cells per well to the upper chambers in $300 \mu 1$ of serum free media. The lower chambers were filled with $500 \mu \mathrm{l}$ of medium containing $10 \%(\mathrm{v} / \mathrm{v})$ fetal bovine serum. After $24-\mathrm{h}$ incubation at $37^{\circ} \mathrm{C}$, non-invading cells were removed from the upper chambers with a cotton-tipped swab. Invading cells in the lower chambers were fixed, stained with $0.5 \%(\mathrm{w} / \mathrm{v})$ crystal violet solution and then lysed using $10 \%(w / v)$ SDS solution. The absorbance at $560 \mathrm{~nm}$ was measured in a microplate reader (Multiskan Ascent, Thermo Electron Corporation, Saint-Herblain, France).

Analysis of proteins. For analysis of PTEN, E-cadherin, vimentin and integrin- $\beta 1$ expression, western blot analysis was realized as previously described (14). The following primary antibodies against human PTEN (M3627, DakoCytomation, Glostrup, Denmark, 1:1,000 dilution), E-cadherin (\#3195, Cell Signaling Technology, Danvers, MA, USA, 1:1,000 dilution), vimentin (\#3390, Cell signaling Technology, 1:1,000 dilution), integrin- $\beta 1$ (\#4706, Cell Signaling Technology,
1:1,000 dilution) and $\beta$-tubulin (sc-9104; Santa Cruz Biotechnology, Santa Cruz, CA, USA, 1:1,000 dilution) were used. Quantification of relative band densities was performed using densitometer (LAS4000 Imager FujiFilm, Tokyo, Japan) and $\beta$-tubulin was used as the internal control.

For analysis of phosphoproteins regulated by PTEN, Bioplex phosphoprotein array based on multiplex sandwich bead immunoassay was used as previously described (14). Fluorescent capturing beads coupled to antibodies directed against phosphorylated-AKT and phosphorylated-ERK 1/2 (Bioplex phosphoprotein assays, Bio-Rad, Marnes-la-Coquette, France) were added into each well containing $6 \mu \mathrm{g}$ of protein.

Screening of soluble proteins secreted by the cells after $48 \mathrm{~h}$ of culture was realized using the proteome profiler array assay (R\&D Systems, Lille, France) as previously described (15). Factors involved in cell invasion (pro-invasive factors, MMP9, uPA; and anti-invasive factors, TIMP1, PAI-1) were detected in significant concentrations.

Tumor growth. All experiments were performed in accordance with national animal care guidelines (European Commission directive 86/609/CEE; French decree no.87-848). Eight-week-old female athymic NMRI-nu (nu/nu) mice (Janvier Laboratories, Saint Berthevin, France) were randomly divided into 4 groups $(n=12)$. All groups received subcutaneous injections of Matrigel and glucose $0.5 \%(1: 3)$ containing $1 \times 10^{7}$ cells. Tumor growth was evaluated, twice a week, by measuring the length and width of the tumor mass with calipers. Tumor volumes were calculated by the modified ellipsoidal formula: $\mathrm{V}=1 / 2$ (length $\mathrm{x}$ width ${ }^{2}$ ).

Histology and immunohistochemistry. Tumor fragments from xenografts harvested 60 days after cell injection were fixed in $4 \%(\mathrm{w} / \mathrm{v})$ formaldehyde, $\mathrm{pH} 7.4$ for $16 \mathrm{~h}$ and embedded in paraffin. Five micrometer sections were used for hematoxylin and eosin and immunochemical staining, as previously described (16). Primary antibodies, sources and dilutions, as well as the antigen retrieval solutions and detection systems of primary antibodies are listed in Table I. Each assay included a negative control consisting of the staining with secondary antibody but in absence of a primary antibody.

Statistical analysis. All results were given as mean \pm standard error of the mean (SEM). Non-parametric Mann-Whitney $\mathrm{U}$ test was employed to determine the statistical significance with a limit set to $\mathrm{p}<0.05$ using GraphPad Prism 5 (GraphPad Software, La Jolla, CA, USA).

\section{Results}

Decrease of PTEN expression activates AKT signaling pathway and promotes cell survival but not cell growth in vitro. We first evaluated the expression of the stable inhibition of PTEN in CAL 27 cell lines (Fig. 1A). We assessed the phosphoproteins expression directly regulated by the phosphatase activity of PTEN, such as pAKT and pERK 1/2. The level of expression of pAKT was decreased by $48 \%$ in the clone shPTEN1, and $38 \%$ in the clone shPTEN2 (Fig. 1B), while the expression of pERK 1/2 was similar to the control cell line (Fig. 1C).

As the semi-quantitative analysis of phospho-proteins was measuring a 3-fold increase in AKT activation in the PTEN 
Table I. Antibodies used for determining protein expression and localization on tissues section.

\begin{tabular}{|c|c|c|c|c|c|}
\hline Antigen & Reference no. & Dilution & Supplier & $\begin{array}{l}\text { Antigen } \\
\text { retrieval } \\
\text { solution }\end{array}$ & Detection system \\
\hline E-cadherin & $\begin{array}{l}\text { Species-unspecific } \\
\text { rabbit } \mathrm{mAB}\end{array}$ & $1 / 600$ & $\begin{array}{l}\text { Cell Signaling } \\
\text { Technology }\end{array}$ & $\begin{array}{l}0.1 \text { MEDTA } \\
\text { pH } 8\end{array}$ & $\begin{array}{l}\text { Biotinylated goat anti-rabbit IgGs/ } \\
\text { streptavidin peroxidase }\end{array}$ \\
\hline Ker 1-3 & $\begin{array}{l}\text { Species-unspecific } \\
\text { mouse } \mathrm{mAB}\end{array}$ & $1 / 300$ & DakoCytomation & $\begin{array}{l}0.1 \mathrm{M} \text { EDTA } \\
\mathrm{pH} 8\end{array}$ & $\begin{array}{l}\text { Biotinylated goat } \mathrm{F}\left(\mathrm{ab}^{\prime}\right) 2 \text { anti-mouse } \operatorname{IgG} 1 / \\
\text { streptavidin peroxidase }\end{array}$ \\
\hline PTEN & $\begin{array}{l}\text { Species-specific } \\
\text { mouse mAB to } \\
\text { human PTEN protein }\end{array}$ & $1 / 150$ & DakoCytomation & $\begin{array}{l}\text { Dako Target } \\
\text { Retrieval } \\
\text { Solution pH } 9\end{array}$ & $\begin{array}{l}\text { Biotinylated goat anti-mouse IgGs/ } \\
\text { streptavidin peroxidase }\end{array}$ \\
\hline Vimentin & $\begin{array}{l}\text { Species-specific } \\
\text { mouse mAB to } \\
\text { human vimetin }\end{array}$ & $1 / 8,000$ & $\begin{array}{l}\text { Cell Signaling } \\
\text { Technology }\end{array}$ & $\begin{array}{l}0.1 \text { M EDTA } \\
\text { pH } 8\end{array}$ & $\begin{array}{l}\text { Biotinylated goat } \mathrm{F}\left(\mathrm{ab}^{\prime}\right) 2 \text { anti-mouse } \operatorname{IgG} 1 / \\
\text { streptavidin peroxidase }\end{array}$ \\
\hline Ki-67 & $\begin{array}{l}\text { Species-specific } \\
\text { rabbit polyclonal } \\
\text { AB to Ki-67 }\end{array}$ & $1 / 100$ & Lab Vision & $\begin{array}{l}0.1 \mathrm{M} \text { EDTA } \\
\mathrm{pH} 8\end{array}$ & $\begin{array}{l}\text { Biotinylated goat anti-rabbit IgGs/ } \\
\text { streptavidin peroxidase }\end{array}$ \\
\hline
\end{tabular}

A

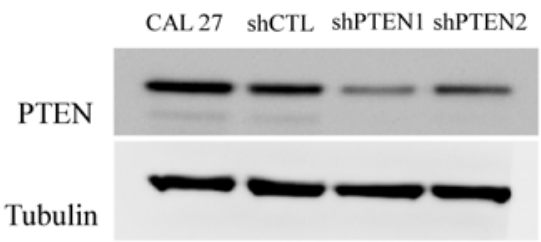

B

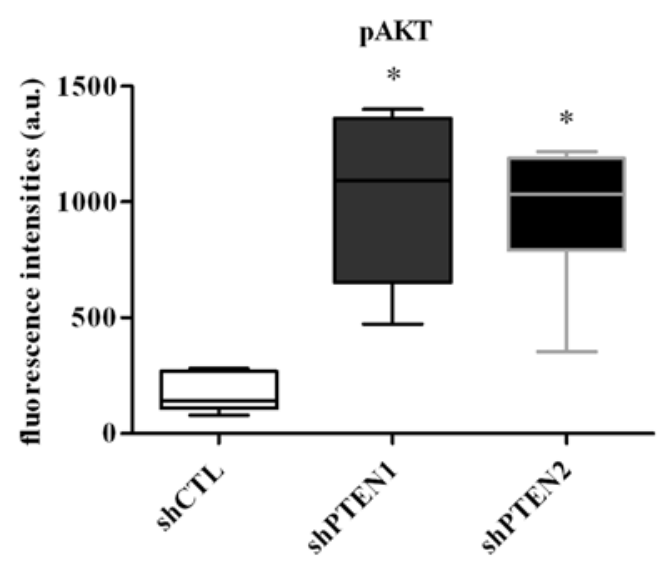

C

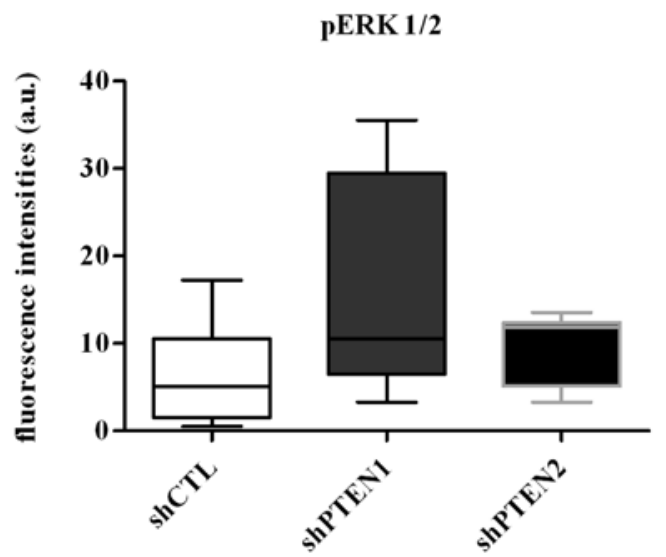

deficient CAL 27 cells, cell growth and survival were assessed by a proliferation assay (Fig. 2). All cell lines were in an exponential phase from 48 to $120 \mathrm{~h}$. The doubling time (calculated on these time points) of shPTEN1 and shPTEN2 was close to those of shCTL and CAL 27 (Table II). Nevertheless a slope change occured at $120 \mathrm{~h}$ (Fig. 2, day 5), when shPTEN1 cells engaged a plateau-like phase, while shPTEN2 cells kept proliferating (day 8) without showing the plateau phase. After this time-point, the number of shCTL and CAL 27 cells was half the number of shPTEN1 and -2 cells (day 10, $\mathrm{p}<0.001$ compared to the shCTL cell line) indicating that cells not only stop proliferating, but also could die. Contrarily, shPTEN cells that were less sensitive to contact inhibition survived and further proliferated. This result is consistent with the overactivation of pAKT, promoting cell survival.

Decrease of PTEN expression is associated with loss of cell adhesion in vitro. In culture, the morphology of PTEN deficient cells suggested a loss of the epithelial phenotype and of cell adhesion. The attachment assay performed at $0.5 \mathrm{~h}$ after plating (Fig. 3A) indicated an interaction of the PTEN-deficient cells significantly increased with specific extracellular matrix (ECM) proteins, notably shPTEN1 cells with fibronectin. This interaction is only transient, as at $4 \mathrm{~h}$, shPTEN cells are not adhering as much compared to the parental and shCTL cell lines (Fig. 3B), notably on laminin and vitronectin. The results suggest that the loss of adhesion was dependent on the inhibition of PTEN, with a loss of adhesion on fibronectin of $18 \%$ in shPTEN1

Figure 1. Inhibition of PTEN expression activates downstream signaling pathways. (A) The expression of PTEN was analyzed by western blot analysis on parental CAL 27, shCTL (CAL 27 cells transfected with a plasmid coding for scramble shRNA and selected with puromycine), shPTEN1 and shPTEN2 (two different colonies, isolated after puromycine selection of CAL 27 cells transfected with a plasmid coding for the same shRNA targeting PTEN mRNA). The expression of (B) pAKT and (C) pERK was assessed on the same lysates, using Bioplex protein array (BPA). BPA results are presented as median of three independent experiments and $\mathrm{p}<0.05$ was considered to be statistically significant. 
Table II. Doubling time of the different CAL 27 cell lines.

CAL 27 ShCTL ShPTEN1 ShPTEN2

Doubling

$\begin{array}{llll}\text { time }(\mathrm{h}) \quad 21.24 \pm 0.91 & 20.54 \pm 1.99 & 19.45 \pm 1.96 & 20.52 \pm 2.26\end{array}$

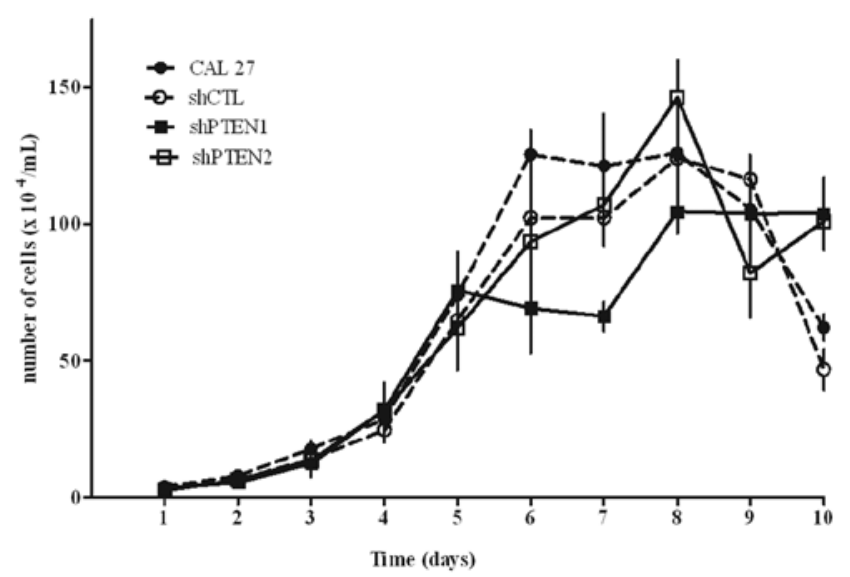

Figure 2. Effect of PTEN silencing on cell growth over 10 days. Proliferation was evaluated in 4 independent trypan blue exclusion assays and presented as concentration over time (cells number x $10^{4} / \mathrm{ml}$ ).

cells and $32 \%$ in shPTEN2, when compared to the shCTL cell line. Associated to the loss of cell adhesion, the expression of $\beta 1$-integrin was found to be upregulated by 25 and $30 \%$, respectively, for shPTEN1 and shPTEN2, as compared to shCTL cells.

Decrease of PTEN expression stimulates cell invasion in vitro. Concomitantly to a loss of adhesion, the increase in $\beta 1$-integrin expression in shPTEN cell lines indicated a gain in mobility. However, a migration assay such as wound healing could not be performed, because shPTEN cells would reach confluency without covering the surface evenly, thus preventing an accurate assessment of wound closure. However, the invasiveness of the cell lines was evaluated using a modified Boyden chamber assay. This assay established that shPTEN1 cells were significantly more invasive than the other cell lines (Fig. 4A), contrary to shPTEN2. In line with this result, the proteolytic proteins MMP9 and UPA were found in higher concentrations in the media of shPTEN1 cells compared to shCTL cells (Fig. 4B). Moreover, TIMP1, inhibitor of MMP9, was not upregulated for shPTEN cells contrary to PAI-1, inhibitor of urokinase-type plasminogen activator (uPA).

As markers of the epithelial to mesenchymal transition, the expression of E-cadherin and vimentin was evaluated in vitro. As expected in epithelial cells, vimentin was not detected, in any of the CAL 27 cell lines. However, E-cadherin expression was significantly different in the PTEN deficient cells, reduced by 2 to 3 times compared to the shCTL cells (Fig. 4C).

Decrease of PTEN expression impairs tumorigenicity of $H N S C C$ cells in vivo. Injected subcutaneously in nude mice, the

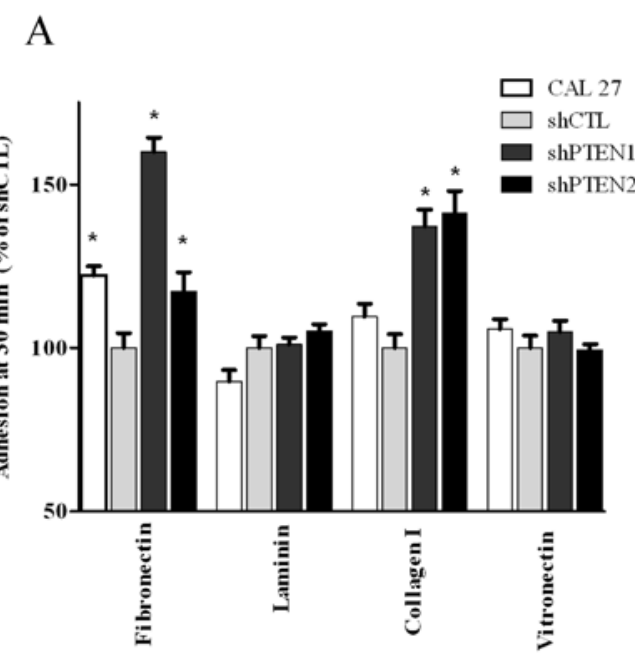

B

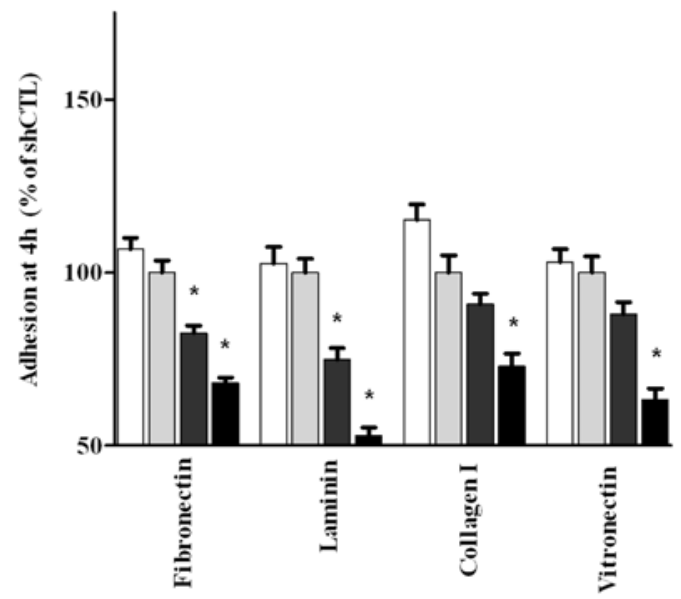

C

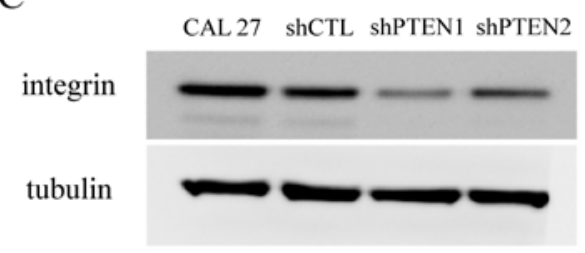

Figure 3. Inhibition of PTEN expression is associated with a loss of cell adhesion and an increase of $\beta 1$ integrin expression. Cell adhesion capacities, depending on PTEN expression in CAL 27 cell lines, were evaluated through the attachment assay onto various ECM proteins: fibronectin, laminin, collagen I and vitronectin, (A) $30 \mathrm{~min}$ and (B) $4 \mathrm{~h}$ after plating. The cells were stained with Hoechst and adhesion measured as fluorescent intensity. The results are presented as a percentage of adhesion to the shCTL cells ( $n=3, p<0.05$ was considered significant). (C) The expression of $\beta 1$ integrin was analyzed by western blot analysis.

parental and shCTL CAL 27 cell lines established tumors within 3 weeks, the tumors of shPTEN cells were palpable but the volume remained smaller than $100 \mathrm{~mm}^{3}$ for 4 weeks (Fig. 5A). Even though there was a significant difference in the growth rate between the shCTL cells and the parental CAL 27 cells, the tumorigenicity of PTEN deficient cells in vivo was significantly reduced. The staining of Ki67 in these tumors confirms that the slow growth is a consequence of a lower proliferation of shPTEN cells (Fig. 5B). 


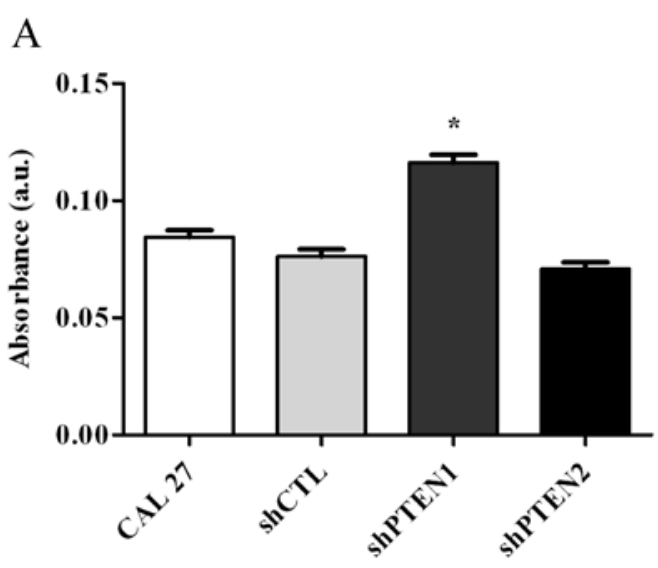

B

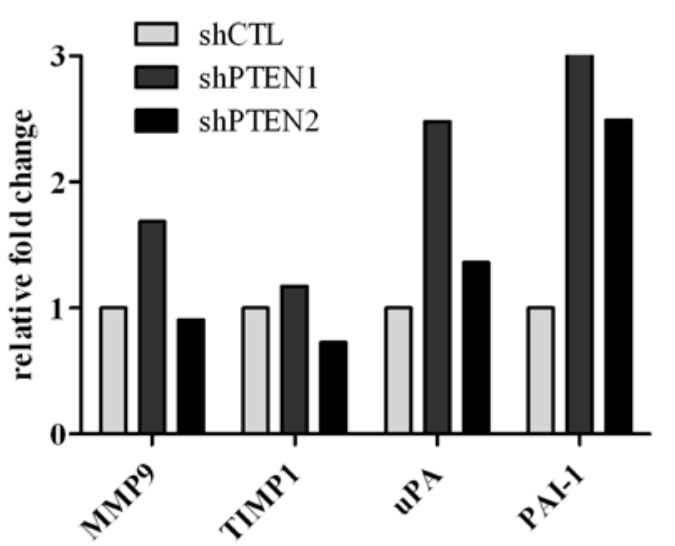

$\mathrm{C}$

CAL 27 shCTL ShPTEN1 ShPTEN2

E-cadherin

Tubulin

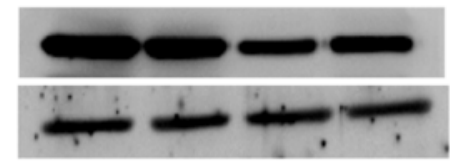

Figure 4. Inhibition of PTEN expression is associated with an increased cell invasiveness and protease expression, while the expression of E-cadherin is downregulated. (A) Cell invasion of the different cell lines was measured using a modified Boyden chamber assay. Cells were fixed and stained with crystal violet, the results of three independent experiments are reported in mean absorbance units. (B) Through a proteome profiler array, secretion of a range of proteases was measured: the change in the amount of MMP9, TIMP1, uPA and PAI-1 in the cell culture media is shown relatively to shCTL. (C) The expression of E-cadherin was analyzed by western blot analysis and a representative blot is shown.

Decrease of PTEN expression induces the formation of islets of well-differentiated tumor cells in vivo. Xenografts of shPTEN cells grew as well-differentiated squamous cell carcinomas with readily apparent keratinization as observed by the cytokeratin staining (arrows on Fig. 6). Some interesting differences can be found in the pattern of staining for PTEN expression. The localization of PTEN in the tumors originating from shCTL cells was predominantly cytoplasmic in epithelial cells. In contrast, the epithelial cells of shPTEN tumors (which still express 40 to $50 \%$ of PTEN protein compared to the shCTL cells) were weakly stained for PTEN, and interestingly the localization was also nuclear for shPTEN1 cells (Fig. 6).
Decrease of PTEN expression is associated with an epithelialmesenchymal transition phenotype in vivo. The expression of molecular markers of EMT: E-cadherin and vimentin, were also evaluated on sections of formalin-fixed tumors (Fig. 7). The membrane E-cadherin staining detected in shCTL and shPTEN1 tumors is typical of an epithelial phenotype (Fig. 7). Interestingly, the staining in shPTEN2 tumors is more diffused. As expected, vimentin was detected in the cytoplasm of the least differentiated cells located at the periphery of the main tumor sheets and cell islets. However, in shPTEN1 tumors, vimentin is stained in the cytoplasm of isolated tumor cells (Fig. 7). This is consistent with the presence of less differentiated territories in these tumors, as well as the presence of numerous isolated tumor cells observed in the connective tissue at a distance of the main tumor masses.

\section{Discussion}

A crucial step in cancer progression is the transforming event turning cells into an overt carcinoma with invasive and metastasic properties. Acquisition of such properties occurs in a multistep and complex process, as cells have to lose adhesion and escape the tumoral environment to disseminate and establish secondary tumors. Epithelial to mesenchymal transition is a modality that can be used by tumors to progress towards dissemination and metastasis, but is still challenging to study in in vivo models or through patient tissue banks (17).

Whereas 50\% of HNSCC patients will suffer from recurrent tumor, new primary tumors or metastasis within 5 years after diagnosis (even after treatment), the mechanisms behind progression have not been elucidated. In a recent review, Leemans et al (18) have described invasion and early metastatic dissemination of HNSCC as a 'biologically-driven' process, highlighting the absence of genes known to induce it. Although the authors review how epithelial to mesenchymal transition could play a role in HNSCC dissemination, notably through the possible implication for the neurotrophic factor receptor NTRK2 and its ligand the brain-derived neurotrophic factor (BDNF), they do not provide any further evidence to substantiate an actual EMT process in HNSCC. This emphasizes the lack of experimental data documenting the process of EMT in HNSCC progression. Our study relates, for the first time, to the common loss of PTEN expression in the epithelial-mesenchymal transition in HNSCC. In our model, the decrease of PTEN expression promoted cell survival in vitro, most likely through an induced activation of AKT, as previously described (14). In addition, the PTEN deficient cells showed a loss of adhesion concomitant to an increased expression of $\beta 1$-integrin, which indicated a gain in migrating properties. Our results also demonstrate that PTEN deficient cells were more invasive than the parental cells, associated to levels of MMP9 and uPA significantly upregulated in shPTEN1 cells. Accordingly, the lower concentration of protease expression detected in shPTEN2 media could explain the discrepancy with shPTEN1 cells in invasiveness. Indeed, uPA-mediated proteolysis was shown to promote tumor invasion, while PAI-1 has been described to compete with integrin binding to vitronectin (19), which is consistent with the data shown here in the adhesion assay.

Together, our results show that the PTEN deficient cells had a survival advantage and were more invasive. Morphological 

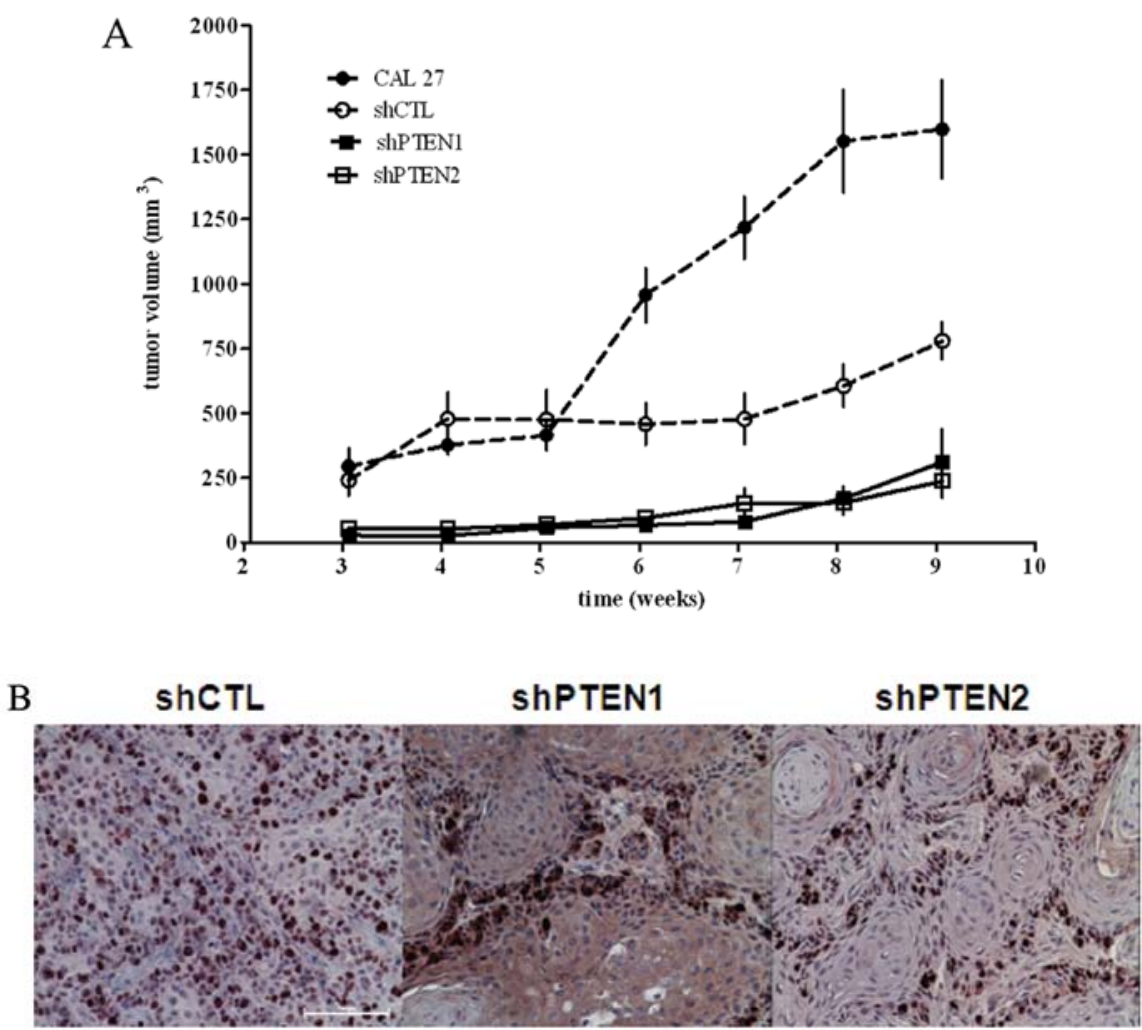

Figure 5. PTEN inhibition impairs tumorigenicity of CAL 27 cells subcutaneously xenografted in nude mice. (A) Tumors growing in the inguinal area over 10 weeks were measured twice a week using a caliper. Results are presented as mean tumor volume, from 2 independent experiments, $\mathrm{n}=12$ tumors. (B) Tumor cell proliferation was evaluated by immunohistological determination of Ki-67 antigen.

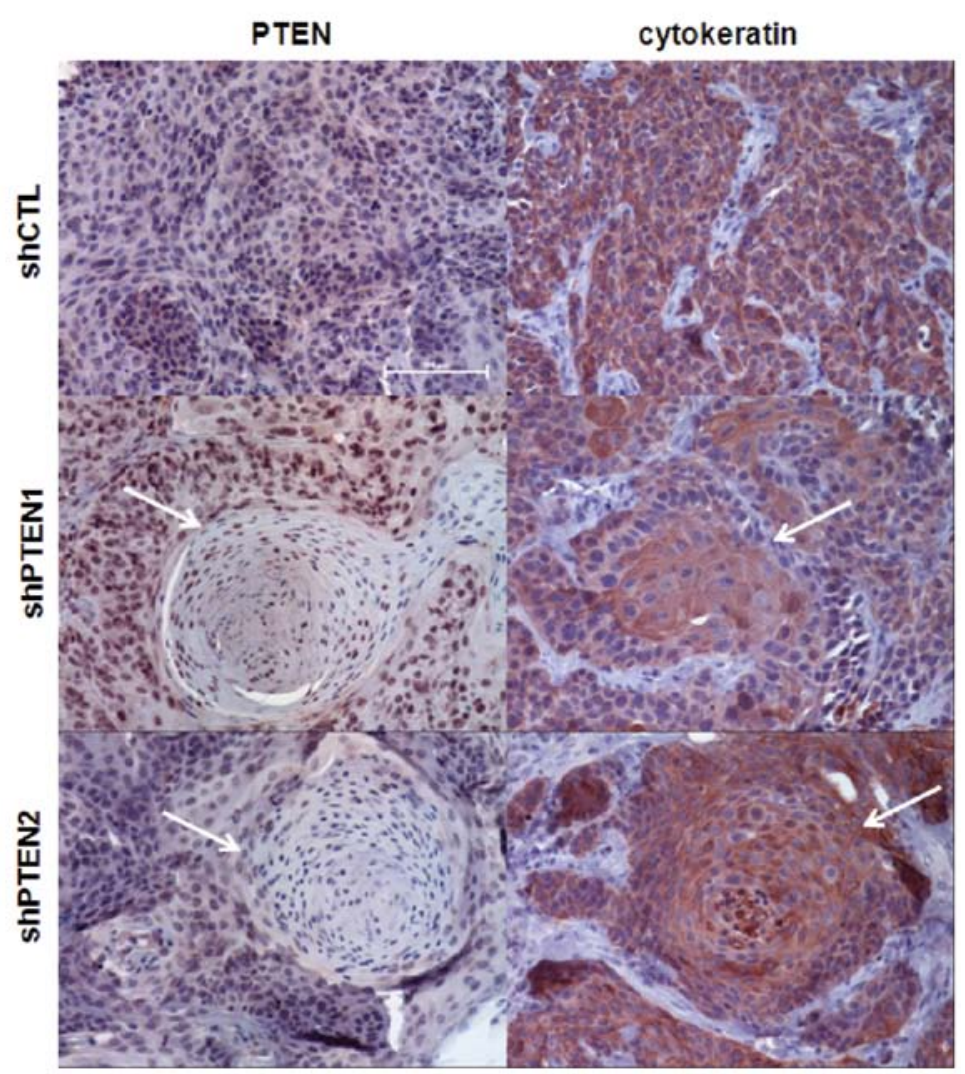

Figure 6. Histological determination of PTEN and cytokeratin expression in tumors derived from shCTL and shPTEN cells. Representative fields of view show the differentiated cell islets (pointed with arrows) on tumors stained for PTEN expression and cytokeratins. The bar scale represents $100 \mu \mathrm{m}$. All stainings were performed on $5 \mu \mathrm{m}$-thick serial sections. 


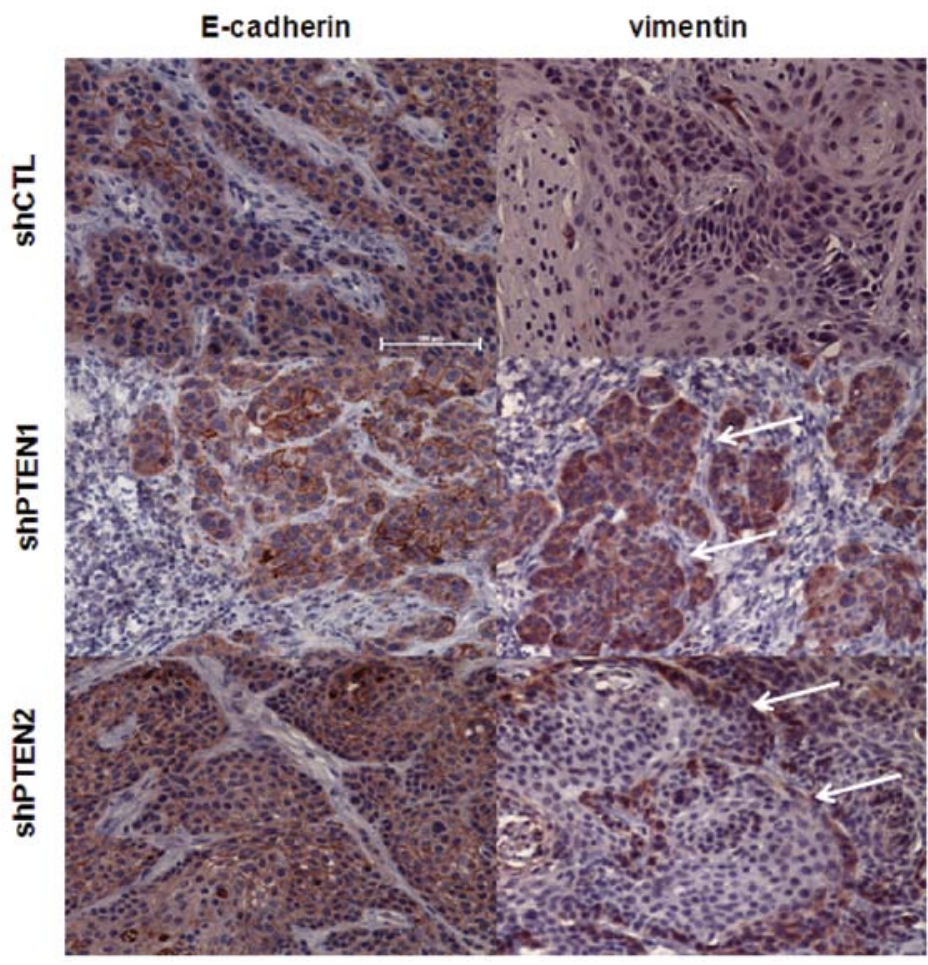

Figure 7. Histological determination of E-cadherin and vimentin expression in tumors derived from shCTL and shPTEN cells. Representative fields of view show the tumors stained for E-cadherin and vimentin expression. Arrows point to vimentin expressing cells. The bar scale represents $100 \mu \mathrm{m}$. All stainings were performed on $5-\mu \mathrm{m}$-thick serial sections.

changes suggested the acquisition of a mesenchymal phenotype, which was confirmed by a decrease in the expression of E-cadherin. Similarly, the morphological observations on sections of formalin-fixed tumors gave some evidence of a diminution of the membrane expression of E-cadherin in PTEN deficient tumors, which also showed the presence of islets of well-differentiated cells. PTEN appears to be essential in maintaining the balance between cell growth, differentiation and malignancy of HNSCC. It has been previously demonstrated that PTEN nuclear localization occurred preferentially in differentiated cells or resting cells (20). Nuclear localization of PTEN may also have distinctive effects on cell growth, apoptosis and differentiation $(21,22)$. Very interestingly, we show that the remaining PTEN protein in tumors originating from shPTEN cells was preferentially localized in the nuclei.

To summarize, our data provide evidence of an epithelial-mesenchymal transition occurring in HNSCC PTEN deficient cells. However, the in vivo model for this study was established with the cells already repressed for PTEN expression, which significantly compromised tumorigenicity. The staining of Ki67 as well as in vitro cell growth showed that it was not due to the proliferation, but could rather be caused by loss of cell adhesion and invasiveness as well as cell differentiation. According to the invasive properties of the PTEN deficient cells, the tumors formed were ulcerative. Interestingly, proliferation in our model, confirmed in vivo, is very similar to the heterozygous loss of PTEN induced in a model of breast cancer cells developed by Vitolo et al (23). The authors show a lower proliferation and a resistance to anoikis (programmed cell death after to cell-detachment of the extracellular matrix).
It would be of interest to evaluate EMT in a conditional knockdown model for PTEN in HNSCC, especially with the loss or reduction of PTEN expression being a late molecular event in the carcinogenesis of HNSCC (24). Similarly, the EMT being a very dynamic process, further live imaging experiments would greatly contribute to elucidating the exact role of PTEN in EMT in this type of cancer.

A link between the expression of PTEN and EMT has been described previously (25), notably through the interaction of PTEN with the $\beta$-catenin/wnt pathway and the downregulation of E-cadherin (26-29). Furthermore, it has been shown that PTEN is involved in stabilization of E-cadherin-mediated adherent junctions (30) and cell polarity (31). We are confident that, with further investigations, PTEN could be proposed as a candidate marker for early dissemination in HNSCC, and possibly the risk of recurrence. Our data are in line with clinical reports $(32,33)$. The most recent one is a genotyping approach conducted on 440 samples from HNSCC patients, in which a genetic variant has been correlated to a high risk of recurrence (33). Similarly, Lee et al (5) already established a link between loss of PTEN expression and advanced disease. Interestingly, in their study, $75 \%$ of the patients with lack of PTEN expression had recurrence, metastasis or death (vs. $41 \%$ in the PTEN positive group).

Our study provides evidence for a new description of role of PTEN in the progression of HNSCC tumors through the EMT. We propose PTEN as a candidate biomarker to predict high risk of recurrence and metastasis in HNSCC. The nuclear localization of PTEN should also be considered in the future histological analysis and may be related to the EMT phenotype. 


\section{Acknowledgements}

The authors thank Professor P. Becuwe for his help in the design of the shRNA plasmid, Professor J.L. Merlin and Ms. Carole Ramacci for their technical help in bioplex protein array and Ms. Dominique Meng for her technical help in immunohistochemistry preparations. The authors also want to thank Dr Desmond Pink for reviewing the English language of this manuscript. This study was supported by grants from the French 'Ligue Nationale contre le Cancer' and the 'Region Lorraine'.

\section{References}

1. Jemal A, Siegel R, Xu J and Ward E: Cancer statistics, 2010. CA Cancer J Clin 60: 277-300, 2010.

2. Mao L, Hong WK and Papadimitrakopoulou VA: Focus on head and neck cancer. Cancer Cell 5: 311-316, 2004.

3. Henderson YC, Wang E and Clayman GL: Genotypic analysis of tumor suppressor genes PTEN/MMAC1 and p53 in head and neck squamous cell carcinomas. Laryngoscope 108: 1553-1556, 1998.

4. Shao X, Tandon R, Samara G, Kanki H, Yano H, Close LG, Parsons R and Sato T: Mutational analysis of the PTEN gene in head and neck squamous cell carcinoma. Int J Cancer 77 : 684-688, 1998.

5. Lee JI, Soria JC, Hassan KA, El-Naggar AK, Tang X, Liu DD, Hong WK and Mao L: Loss of PTEN expression as a prognostic marker for tongue cancer. Arch Otolaryngol Head Neck Surg 127: 1441-1445, 2001.

6. Kwon CH, Zhao D, Chen J, Alcantara S, Li Y, Burns DK, Mason RP, Lee EY, Wu H and Parada LF: Pten haploinsufficiency accelerates formation of high-grade astrocytomas. Cancer Res 68: 3286-3294, 2008.

7. Alimonti A, Carracedo A, Clohessy JG, Trotman LC, Nardella C, Egia A, Salmena L, Sampieri K, Haveman WJ, Brogi E, Richardson AL, Zhang J and Pandolfi PP: Subtle variations in Pten dose determine cancer susceptibility. Nat Genet 42: 454-458, 2010

8. Vazquez F and Sellers WR: The PTEN tumor suppressor protein: an antagonist of phosphoinositide 3-kinase signaling. Biochim Biophys Acta 1470: 21-35, 2000.

9. Gu J, Tamura M and Yamada KM: Tumor suppressor PTEN inhibits integrin- and growth factor-mediated mitogenactivated protein (MAP) kinase signaling pathways. J Cell Biol 143: 1375-1383, 1998.

10. Dey N, Crosswell HE, De P, Parsons R, Peng Q, Su JD and Durden DL: The protein phosphatase activity of PTEN regulates SRC family kinases and controls glioma migration. Cancer Res 68: 1862-1871, 2008.

11. Tamura M, Gu J, Danen EH, Takino T, Miyamoto S and Yamada KM: PTEN interactions with focal adhesion kinase and suppression of the extracellular matrix-dependent phosphatidylinositol 3-kinase/Akt cell survival pathway. J Biol Chem 274: 20693-20703, 1999.

12. Haier $\mathbf{J}$ and Nicolson GL: PTEN regulates tumor cell adhesion of colon carcinoma cells under dynamic conditions of fluid flow. Oncogene 21: 1450-1460, 2002.

13. Bowen KA, Doan HQ, Zhou BP, Wang Q, Zhou Y, Rychahou PG and Evers BM: PTEN loss induces epithelial-mesenchymal transition in human colon cancer cells. Anticancer Res 29: 4439-4449, 2009.

14. Mriouah J, Boura C, Pinel S, Chretien AS, Fifre A, Merlin JL and Faivre B: Cellular response to cetuximab in PTEN-silenced head and neck squamous cell carcinoma cell line. Int J Oncol 37: 1555-1563, 2010.

15. Jouan-Hureaux V, Boura C, Merlin JL and Faivre B: Modulation of endothelial cell network formation in vitro by molecular signaling of head and neck squamous cell carcinoma (HNSCC) exposed to cetuximab. Microvasc Res 83: 131-137, 2012.

16. Labussière $M$, Pinel $S$, Vandamme $M$, Plénat $F$ and Chastagner $P$. Radiosensitizing properties of bortezomib depend on therapeutic schedule. Int J Radiat Oncol Biol Phys 79: 892-900, 2011.
17. Thiery JP, Acloque H, J. Huang RY and Nieto MA: Epithelialmesenchymal transitions in development and disease. Cell 139: 871-890, 2009.

18. Leemans CR, Braakhuis BJ and Brakenhoff RH: The molecular biology of head and neck cancer. Nat Rev Cancer 11: 9-22, 2011.

19. Durand MK, Bodker JS, Christensen A, Dupont DM, Hansen M, Jensen JK, Kjelgaard S, Mathiasen L, Pedersen KE, Skeldal S, Wind T and Andreasen PA: Plasminogen activator inhibitor-I and tumour growth, invasion, and metastasis. Thromb Haemost 91: 438-449, 2004

20. Gimm O, Perren A, Weng LP, Marsh DJ, Yeh JJ, Ziebold U, Gil E, Hinze R, Delbridge L, Lees JA, Mutter GL, Robinson BG, Komminoth P, Dralle H and Eng C: Differential nuclear and cytoplasmic expression of PTEN in normal thyroid tissue, and benign and malignant epithelial thyroid tumors. Am J Pathol 156: 1693-1700, 2000.

21. Liu JL, Sheng X, Hortobagyi ZK, Mao Z, Gallick GE and Yung WK: Nuclear PTEN-mediated growth suppression is independent of Akt down-regulation. Mol Cell Biol 25: 6211-6224, 2005.

22. Ginn-Pease ME and Eng C: Increased nuclear phosphatase and tensin homologue deleted on chromosome 10 is associated with G0-G1 in MCF-7 cells. Cancer Res 63: 282-286, 2003.

23. Vitolo MI, Weiss MB, Szmacinski M, Tahir K, Waldman T, Park BH, Martin SS, Weber DJ and Bachman KE: Deletion of PTEN promotes tumorigenic signaling, resistance to anoikis, and altered response to chemotherapeutic agents in human mammary epithelial cells. Cancer Res 69: 8275-8283, 2009.

24. Braakhuis BJ, Brakenhoff RH and Leemans CR: Treatment choice for locally advanced head and neck cancers on the basis of risk factors: biological risk factors. Ann Oncol 23: 173-177, 2012.

25. Wang H, Quah SY, Dong JM, Manser E, Tang JP and Zeng Q: PRL-3 down-regulates PTEN expression and signals through PI3K to promote epithelial-mesenchymal transition. Cancer Res 67: 2922-2926, 2007.

26. Craene BD and Berx G: Regulatory networks defining EMT during cancer initiation and progression. Nat Rev Cancer 13: 97-110, 2012

27. Li Z, Wang L, Zhang W, Fu Y, Zhao H, Hu Y, Prins BP and Zha X: Restoring E-cadherin-mediated cell-cell adhesion increases PTEN protein level and stability in human breast carcinoma cells. Biochem Biophys Res Commun 363: 165-170, 2007.

28. Lau MT, Klausen C and Leung PC: E-cadherin inhibits tumor cell growth by suppressing PI3K/Akt signaling via $\beta$-cateninEgrl-mediated PTEN expression. Oncogene 30: 2753-2766, 2011.

29. Fournier MV, Fata JE, Martin KJ, Yaswen P and Bissell MJ: Interaction of E-cadherin and PTEN regulates morphogenesis and growth arrest in human mammary epithelial cells. Cancer Res 69: 4545-4552, 2009.

30. Kotelevets L, van Hengel J, Bruyneel E, Mareel M, van Roy F and Chastre E: Implication of the MAGI-1b/PTEN signalosome in stabilization of adherens junctions and suppression of invasiveness. FASEB J 19: 115-117, 2005.

31. Langlois MJ, Bergeron S, Bernatchez G, Boudreau F, Saucier C, Perreault N, Carrier JC and Rivard N: The PTEN phosphatase controls intestinal epithelial cell polarity and barrier function: role in colorectal cancer progression. PLoS One 5: e15742, 2010.

32. Snietura M, Jaworska M, Mlynarczyk-Liszka J, Goraj-Zajac A, Piglowski W, Lange D, Wozniak G, Nowara E and Suwinski R: PTEN as a prognostic and predictive marker in postoperative radiotherapy for squamous cell cancer of the head and neck. PLoS One 7: e33396, 2012.

33. Hildebrandt MA, Lippman SM, Etzel CJ, Kim E, Lee JJ, Khuri FR, Spitz MR, Lotan R, Hong WK and Wu X: Genetic variants in the PI3K/PTEN/AKT/mTOR pathway predict head and neck cancer patient second primary tumor/recurrence risk and response to retinoid chemoprevention. Clin Cancer Res 18: 3705-3713, 2012 